\title{
Atrial Activity Extraction in Holter Registers using Adaptive Wavelet Analysis
}

\author{
C Sánchez ${ }^{1}$, J J Rieta ${ }^{2}$, F Castells ${ }^{2}$, J Ródenas ${ }^{1}, \mathrm{~J} \mathrm{Millet}^{2}$ \\ ${ }^{1}$ DIEEA. E.U.P. Cuenca, Universidad de Castilla-La Mancha, Spain \\ ${ }^{2}$ BET. DIEo. Universidad Politécnica Valencia, Spain
}

\begin{abstract}
Extraction of atrial activity $(A A)$ is quite important in the study of different atrial arrhythmias. This work shows the possibility of $A A$ extraction from Atrial Fibrilation $(A F)$ episodes in Holter registers using only one lead with a new technique, the adaptive wavelet analysis (AWA). The principal aim is to adapt automatically the Discrete Packet Wavelet Transform (DPWT) depending on the shape of the signal considered in each moment. The more suitable wavelet functions are selected to obtain several ideal wavelet forms. Every register is divided in different blocks and a DPWT is applied using the corresponding wavelet in each block. So, the analysis is adapted to the behaviours and properties of the lead under analysis. After the mentioned process, the AA is reconstructed using the best coefficients of the obtained wavelet decomposition. The AWA should be applicable in arrhythmia detection and analysis, like paroxismal atrial fibrillation, which have to be usually detected from Holter systems.
\end{abstract}

\section{Introduction}

Atrial Fibrillation (AF) is one of the most common arrhythmias. About the $2-4 \%$ of the people above 60 years suffer from AF. These numbers raise by $12 \%$ in people above 75 [1]. The paroxysmal AF is one type of AF, it appears in episodes with lengths below 48 hours and in most cases have to be detected in Holter registers where the number of leads is reduced. The isolated study of the registered atrial activity (AA) in the electrocardiogram (ECG) is necessary for the detection and characterization of the AF in these cases [2-4]. This study requires a previous extraction or cancellation of the ventricular activity (VA) which is spectrally overlapped and has larger amplitude level than the AA.

In previous works [5], the Discrete Packet Wavelet Transform (DPWT) has been applied as a non-invasive method for AA extraction. The main advantage of the analysis with DPWT is the needles of several leads from the ECG, hence, it is ideal for the study of Holter registers, where the number of available leads is reduced. The main problem of DPWT is the poor performance in real $\mathrm{AF}$ registers under extreme chaotic behaviours what make necessary the use of adaptive systems [6-8]. One of the main problem and, at the same time, goals of the present wavelet-based study is to find the more suitable wavelet mother function. In AF registers, the base line fluctuations (known as ' $\mathrm{f}$ ' waves) and the fast changes in size, orientation and length of these waves make difficult the election of one wavelet function for the whole analysed segment.

In this work, the Adaptive Wavelet Analysis (AWA) is presented as a new method to extract the AA. It is based on the same methodology as the DPWT but, now, the AWA is able to select and change to the more suitable wavelet family, in different fragments of the ECG according with the form of each QRS complex. This technique improves considerably the results obtained by previous works using only a function in the whole $\mathrm{AF}$ episode.

\section{Methods}

\subsection{Database}

A database of artificial signals has been created, where the AA is known and simulated. A synthesized AF signal has been added to sinus rhythm recordings using linear combination, so the shape and behavior of the atrial signal is known and the performance of the method can be measured by comparison of expected and estimated AA. These AF episodes present a dominant peak in 6.10 $\mathrm{Hz}$ and a $70 \%$ spectral energy concentration around this peak. These are typical values found in the spectral analysis of real AF recordings [9]. The signals used are created from recordings of the MIT-BIT and an own database of ECG, with signals obtained at the Cardiac Electrophysiology Lab of the University Clinical Hospital in Valencia and diagnosed by cardiologists. This database contains sinus rhythms, real AF episodes and artificial AF episodes. The length of all the registers is 5 seconds, the sample rate is $1 \mathrm{kHz}$, and $\mathrm{V} 1$ is the considered lead. All the registers have been pre-processed and normalized to remove possible fluctuations of the base line, interferences, noises, etc. The configuration of the database is shown in Table 1. 
Table 1. Registers used in the database.

\begin{tabular}{lll}
\hline & Synthesized SR+AF & Real AF \\
\hline Number & 19 & 8 \\
\hline
\end{tabular}

\subsection{Wavelet analysis}

Wavelet analysis consists of decomposing a signal, in this case the ECG signal, into a hierarchical set of wavelets coefficients; approximations and details. These coefficients are obtained from the signal multiplied by scaled -compressed or stretched- and shifted -delayed or hastened- versions of the mother wavelet function $\Psi$. The set of translated and scaled wavelets associated with $\Psi$ can be expressed as follows, where the parameters ' $a$ ' and ' $b$ ' determine the scale and position respectively.

$$
\frac{1}{\sqrt{a}} \psi\left(\frac{x-b}{a}\right)
$$

In the Discrete Wavelet Transform (DWT) only the approximation coefficients are split in consecutive approximation and detail coefficients. However, in the DPWT, the details as well as the approximations can be split. This offers too much information and it requires to find the best signal decomposition tree. Therefore, DPWT can be considered as a generalization of the DWT and it is recommended in applications where the behaviour of the analysed signal can change quickly being necessary to use different decomposition trees in different fragments of the same signal, as occurs in our case. There are different types of wavelet families whose qualities vary according to several criteria. The wavelet decomposition could be not the optimum if a suitable family is not chosen.

\subsection{Adaptive wavelet analysis}

The basic idea in this study is to detect the QRS complexes of the considered lead in a first stage, using traditional methods of peak detection and windowing [10]. After that, the estimated form of each located QRS complex is compared with a data base of wavelet functions and a set of cross correlation coefficients are obtained. This database is created with scaled and shifted versions of a selected group of typical wavelet families. Figure 1 and Figure 2, show the form of some of these functions.

The greatest values of cross correlation coefficients indicate the most suitable function and the best level of decomposition for each selected fragment. In a second stage, an adaptive DPWT is done using those functions and levels, the VA is located in a set of coefficients in the decomposition tree and the AA can be extracted.
Finally, all the segments are joined using linear interpolation to reduce possible border effects and the AA is reconstructed in the complete cardiac cycle.

In some registers, the use of another consecutive DWT in the obtained signal has been necessary to remove possible residual QRS complexes of the previous stages. In these cases, the Daubechies 10 function has been used for the whole signal.

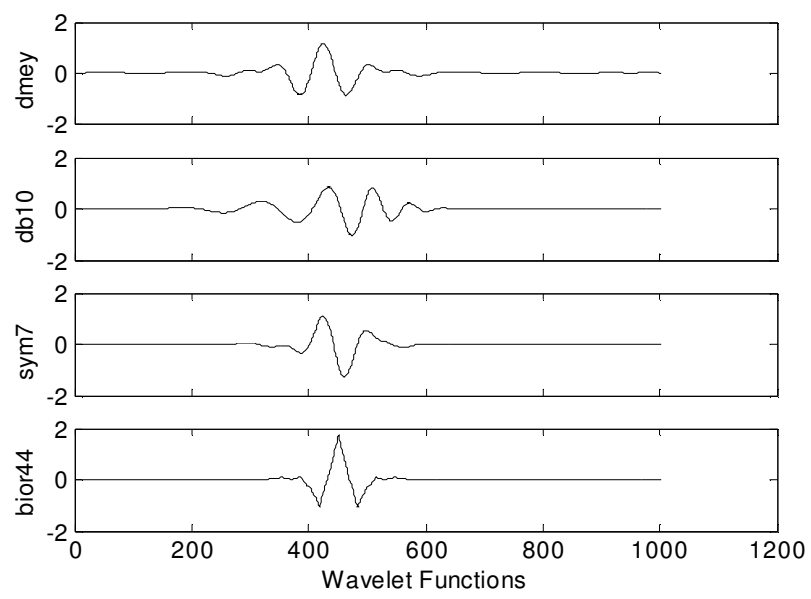

Figure 1. Different forms of typical wavelet families in the same level of decomposition.

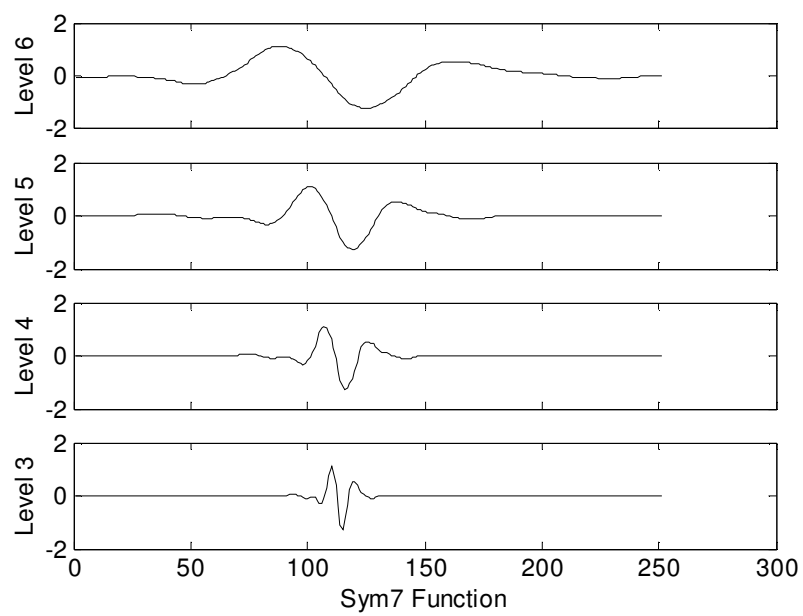

Figure 2. Different scaled levels of a typical wavelet family: Symlets 7.

\section{Results}

In synthesized AF signals, the comparison between the obtained signal and the expected one is done with the cross correlation coefficients in the spectral and temporal domain. With respect to real AF signals, additional parameters have been considered to identify the AF episode, for example, principal peak and spectral energy concentration in the band of $5-8 \mathrm{~Hz}$ (named as SCBP-AA in the table of results). The high energy concentration in this band in respect of the total energy is typical in AF 
episodes that present a dominant peak in this range. An increase of this concentration involves a greater purity of the extracted AA and it is considered as quality extraction index [11]. The AWA and DPWT methods have been applied to the registers described in Table 1. The comparison among the obtained results are shown in Table 2 for the synthesized AF episodes and in Table 3 for the real AF episodes, including their mean value and standard deviation $(\mathrm{p} \leq 0.05)$.

Table 2. Correlation coefficients obtained with synthesized AF episodes.

\begin{tabular}{lcc}
\hline & DPWT & AWA \\
\hline Spectral Corr. & $0.84 \pm 0.11$ & $0.91 \pm 0.08$ \\
\hline Temporal Corr. & $0.52 \pm 0.17$ & $0.61 \pm 0.11$ \\
\hline
\end{tabular}

Table 3. Spectral parameters obtained with real AF episodes.

\begin{tabular}{lcc}
\hline & DPWT & AWA \\
\hline Main Peak $(\mathrm{Hz})$ & $6.15 \pm 0.37$ & $6.03 \pm 0.21$ \\
\hline SCBP-AA & $0.62 \pm 0.19$ & $0.76 \pm 0.12$ \\
\hline
\end{tabular}

As it can be observed, the AWA improves the quality extraction of the AA in all the cases. In real signals, the detected main peak is basically the same in both methods but the values of SCBP-AA are clearly higher in AWA.
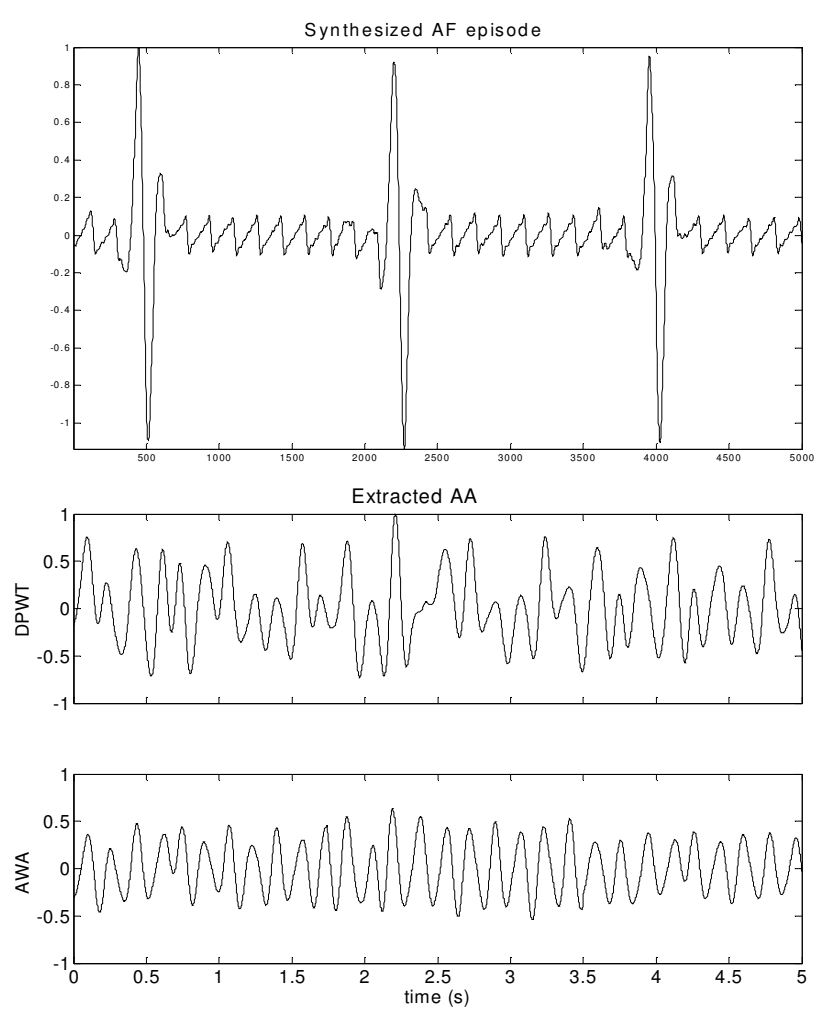

Figure 3. Comparison of extracted AA in synthesized signals.
In Figure 3, it is shown the form of the extracted AA from a synthesized AF register of the database. There are not residual complexes in any case but the signal obtained with the DPWT shows fluctuations, not present in the original AA and in the AWA signal. This fact has been observed in the rest of the registers, being this one of the causes of the poor performance of DPWT.

Figure 4 shows the results in the case of real AF episodes.
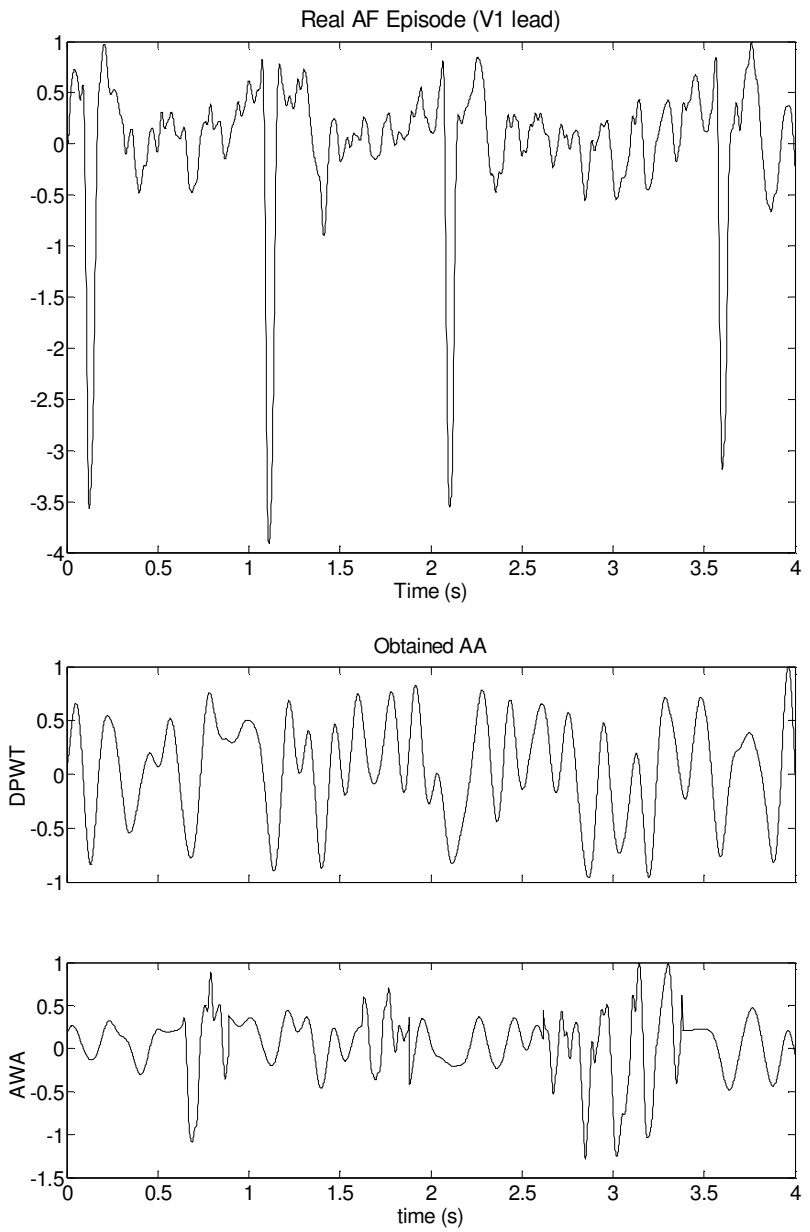

Figure 4. Comparison of extracted AA in real AF episodes.

The form of the AA achieved from DPWT does not respect the original shape of the ' $f$ ' waves and it is very similar to a sum of sinusoids. Although some residuals sinusoids of the same type appear in the place where the QRS complexes are located, this distortion is reduced with the use of AWA, .

Figure 5 shows the spectral distributions of the different obtained AA, where frequencies above $35 \mathrm{~Hz}$ have not been considered. The parameters used to calculate this distributions are the Welch method, Bartlett window of 4096 points, 50\% overlapping and a 8192 points FFT. 

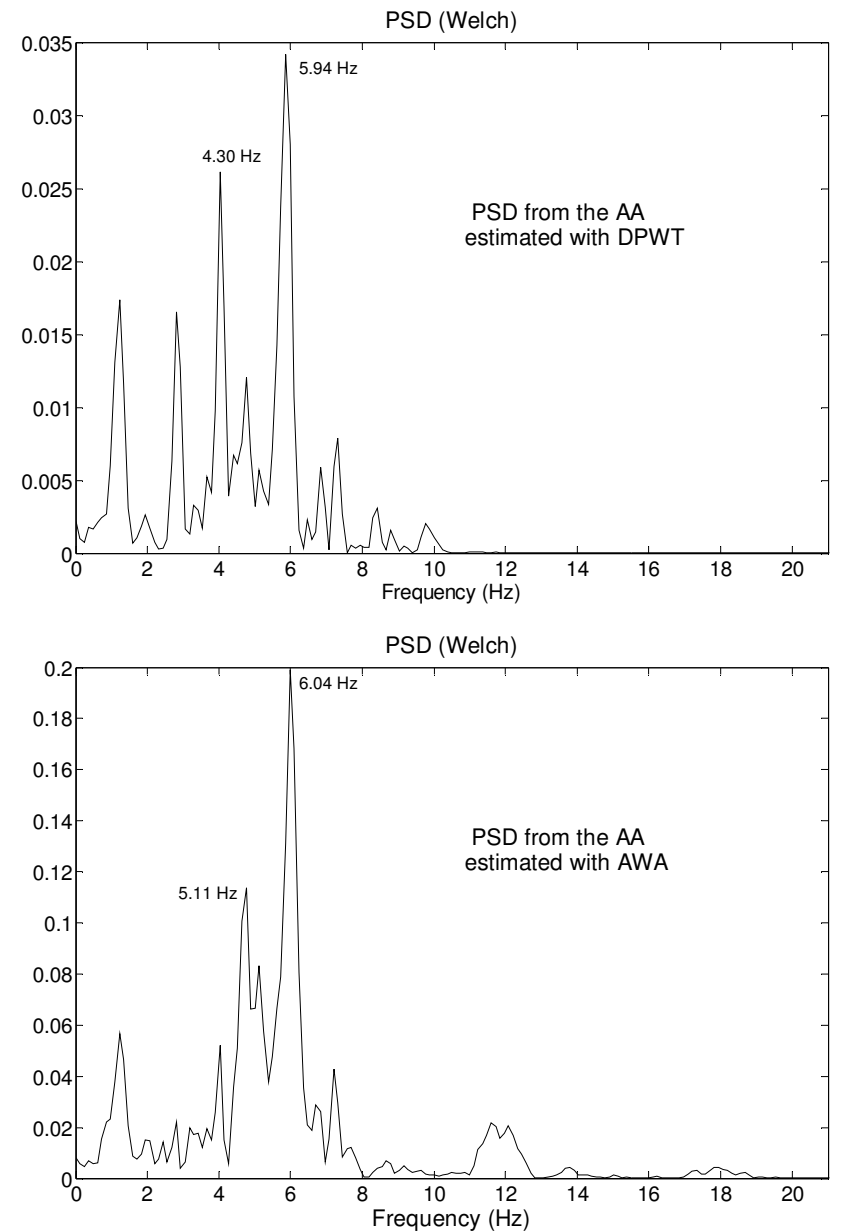

Figure 5. Comparison of PSD estimated in extracted AA from real $\mathrm{AF}$ registers with both methodologies.

The greater concentration of the energy in the case of AWA demonstrates a better quality of the extraction, as it can be observed.

\section{Conclusion}

Throughout this work, the improvements of the AWA as a complement of the DPWT have been shown. This is an important step to find an atrial extraction method applicable in short duration registers with a reduced number of leads. The presented adaptive process makes the system more reliable in the cases of AF episodes with fast fluctuations and abnormal beats where other methods are less efficient.

Finally, this complementary process, joined to DPWT methods, should be applicable in arrhythmia detection and analysis, like paroxismal atrial fibrillation, which have to be usually detected from Holter systems.

\section{Acknowledgements}

This work was partly funded by several research grants from the D.I.E.E.A. in the University of CastillaLa Mancha, the TIC2002-00957 and CTIDIA/202/053. The authors would like to thank cardiologists R. Ruiz, S. Morell, F.J. Chorro and R. Garcia from the University Clinical Hospital of Valencia, for their clinical advices and kind help in obtaining the signals.

\section{References}

[1] Falk RH. Medical progress: Atrial fibrillation. New England Journal of Medicine 2001; 344(14):1067-1078.

[2] Rieta JJ, Castells F, Sanchez C, Igual J. ICA applied to atrial fibrillation analysis. International Conference on Independent Component Analysis and Blind Signal Separation (ICA) 2003; 4:59-64.

[3] Stridh M, Sörnmo L. Spatiotemporal QRST cancellation techniques for analysis of atrial fibrillation. IEEE Trans Biomed Eng 2001;48(1):105-111.

[4] Langley P, Stridh M, Rieta JJ, Sörnmo L, Millet-Roig J, Murray A. Comparison of Atrial Rhythms Extraction Techniques for the Estimation of the Main Atrial Frequency from the 12-lead Electrocardiogram in Atrial Fibrillation . IEEE Computers in Cardiology 2002;29:29-32.

[5] Sánchez C, Millet J, Rieta JJ, Ródenas J, Castells F. Packet Wavelet Decomposition: An Approach to Atrial Activity Extraction. IEEE Computers in Cardiology 2002;29: 33-36.

[6] Shkurovich S, Sahakian AV, Swiryn S. Detection of atrial activity from high-voltage leads of implantable ventricular defibrillators using a cancellation technique. IEEE Trans Biomed Eng 1998;45(2):229-234.

[7] Thakor N, Yi-Zheng Z. Applications of adaptative filtering to ECG analysis: Noise cancellation and arrhytmia detection. IEEE Trans. Biomed Eng. 1991; 48(8):785-794.

[8] Colomer A, Terré V. Electrocardiogram enhancement by adaptative filtering. Signal Processing VII: Theories and Applications (EUSIPCO) 1994;1:58-61.

[9] Millet, J., Rieta, J. J., Zarzoso, V., Cebrian, A., Castells, F., Sanchez, C., and Garcia, R. Surface-ECG Atrial Activity Extraction via Blind Source Separation: Spectral Validation. IEEE Computers in Cardiology 2002;29:605608.

[10] Kadambe s, Murray R, Fayé Bouderaux-Bartells G. Wavelet Transform-Based QRS Complex Detector. IEEE Trans. Biomed Eng. 1999; 46:838-848.

[11] Castells F, Ruiz R, Rieta JJ, Millet J. An integral atrial wave identification approach based on spatiotemporal source separation: Clinical validation. IEEE Computers in Cardiology 2003;30:In press. Thessaloniki, Greece.

Address for correspondence César Sánchez Meléndez E.U.P. Cuenca. Universidad de Castilla-La Mancha. Campus Universitario s/n. 16071. Cuenca.

E-mail: cesar.sanchez@uclm.es 